\title{
Strategies for Visualization of Extracellular Polymeric Substances (ExPS) in Biofilms by Electron Microscopy
}

\author{
Alice C. Dohnalkova ${ }^{1}$, Bruce W. Arey ${ }^{1}$, Kenneth H. Williams ${ }^{2}$ and Matthew J. Marshall ${ }^{1}$ \\ ${ }^{1}$ Pacific Northwest National Laboratory, Richland, WA, ${ }^{2}$ Lawrence Berkeley National \\ Lab, Berkeley, CA
}

Microbial-metal interaction in bacterial biofilms in the subsurface play an important role that can be used for developing bioremediation technologies for environmental cleanup of soils contaminated with heavy metals and radionuclides. Dissimilatory metal reducing bacteria (DMRB) are able to utilize a wide range of substrates as an electron acceptor for their anaerobic respiration, resulting in reduction and thus immobilization of multivalent metals such as $\mathrm{U}, \mathrm{Cr}$ and Tc. In our previous communication [1], we identified the role of c-type cytochromes localized in extracellular polymeric substances (ExPS) during the direct metal reduction by electron transfer and their sites of reduced metal deposition in Shewanella oneidensis MR-1. Since the ExPS play an important role in the metal reduction, a thorough morphological description in regards of the cell-mineral association is needed for accurate interpretations of microbial-metal reduction processes.

We studied a biofilm sample obtained from a borehole used for long term (100+day) acetate injection during biostimulation activities at DOE's Rifle Integrated Field Research Challenge (IFRC) site near Rifle, Colorado. The geochemical conditions surrounding formation of the biofilm included stimulated iron and sulfate reduction, which led to the accumulation of polymer-associated ferrous iron minerals, such as $\mathrm{FeS}$ and $\mathrm{FeCO} 3$, as well as calcite. After their collection, the mineral encrusted biofilms and ExPS were preserved in geochemically reduced, sulfidic groundwater pumped from the sampling borehole before shipment to the microscopy facility.

In order to assess properties of the biofilm and ExPS under in situ hydrated conditions, a variety of EM imaging methods were used. We evaluated them on the merit of direct and indirect visualization of cellular and ExPS features, and aptness for the newly formed minerals analyses. The extremely hydrated extracellular polysaccharides pose a serious challenge with the dehydration that is a pre-requisite for the vacuum-based electron microscopy. After the fixation, samples underwent a series of ExPS stabilization or labeling, then were dehydrated in ethanol series, and either plastic embedded for TEM or processed for SEM. Namely, ruthenium red that specifically binds to anionic sites was used as a tracer of carbohydrates on the cell surface and in ExPS. The other - indirect ExPS visualization was done by labeling with Concanavalin A (ConA) conjugated with $10 \mathrm{~nm}$ colloidal gold. As a lectin, ConA has affinity for specific sugars sequences in oligosaccharide residues present in ExPS. A good amount of labeling was achieved, but without post-staining, the ExPS was practically invisible. We try to avoid the poststaining since the substantial volume of this research studies uranium (VI) reduction; thus the classical uranyl acetate/lead citrate must be excluded. Both Ru red and ConA treatment were evaluated by TEM (plastic embedding, thin sections) and SEM (dehydration, CPD or only air drying). Air dried Ru red samples seemed to have the ExPS stabilized better than the critical point dried (CPD). CryoEM was explored for 
better preservation of the ExPS integrity in fully or partially hydrated conditions. CryoSEM showed more cellular and ExPS detail after partial sublimation. CryoTEM of frozen-hydrated sections, besides requiring a very labor-intensive preparation, showed a very good cell morphology and close associations, however the ExPS was virtually invisible due to the lack of electron dense stain.

In conclusion, a suite of correlative EM methods should be used for visualization of bacterial interactions with metals and accompanied by analytical methods such as energy dispersive spectrosopy (EDS) for elemental composition and selected area diffraction for crystalolographic information.

\section{[1] Marshall MJ et al., PLoS Biology 4:1324-33 (2006)}

This research was performed using EMSL, a national scientific user facility sponsored by the Department of Energy's Office of Biological and Environmental Research located at Pacific Northwest National Laboratory.

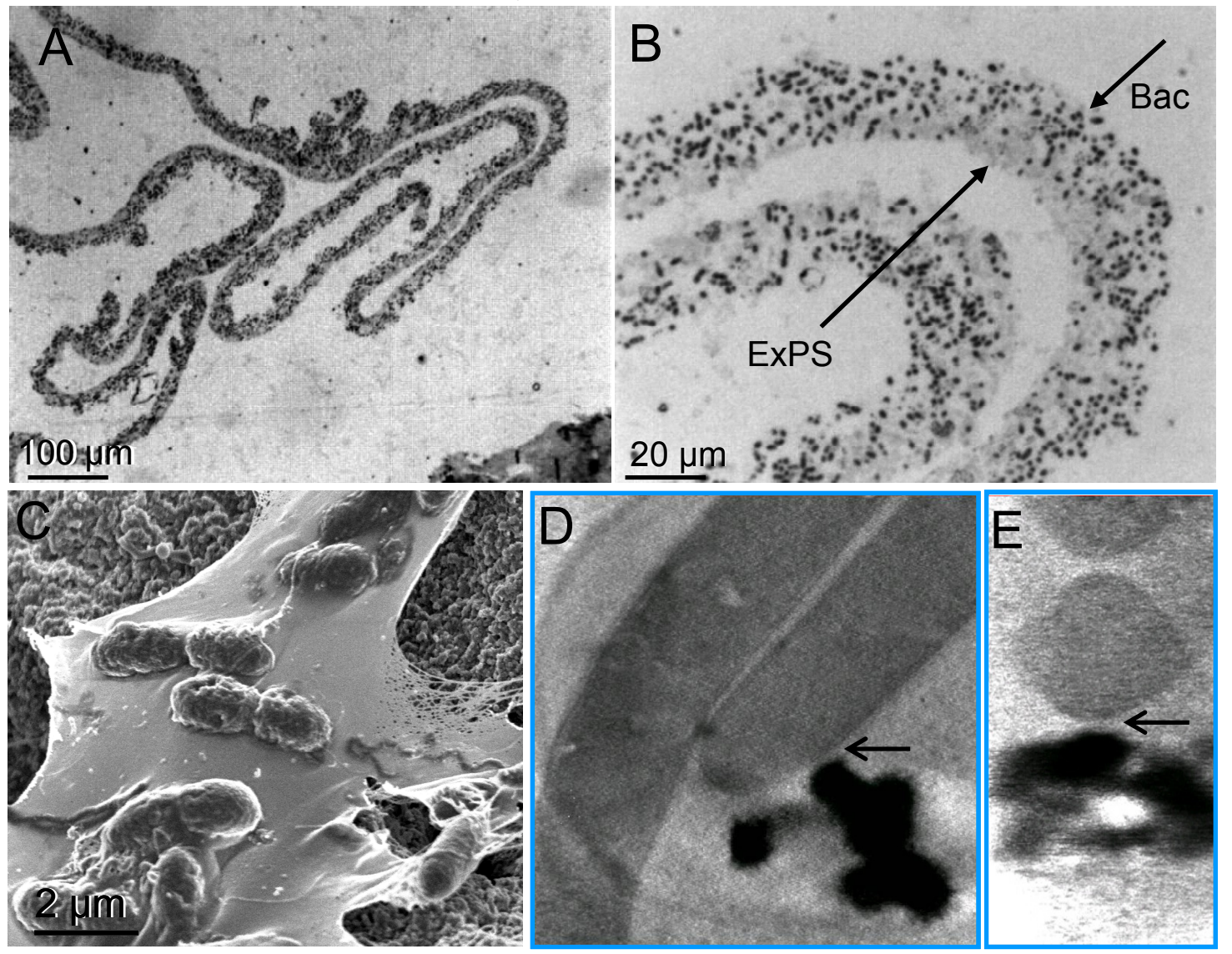

Fig 1. Light micrographs of a semi-thin plastic section of a biofilm layer (pellicle) $(A, B)$. A cross section shows morphological heterogeneity that influences the metal reduction gradient across the biofilm. SEM image of Ru-red treated biofilm shows improved stabilization of ExPS (C). CryoTEM tomographic slices from the $3 \mathrm{~d}$ reconstruction in the $z$ and $x$ direction (D,E, resp.) show cells keeping $\sim 30 \mathrm{~nm}$ distance from the mineral substrate. Although with no electron contrast for direct imaging, ExPS is believed to be present to serve as a mediator of the electron transfer between the cell and a mineral. 